\title{
Direct Observation of Domains and Discommensurations in Mn-Si-Al Octagonal Quasicrystal
} by Transmission Electron Microscopy

\author{
J. C. Jiang, ${ }^{(1),(2)}$ N. Wang, ${ }^{(1),(2)}$ K. K. Fung, ${ }^{(1),(3)}$ and K. H. Kuo ${ }^{(1),(2)}$ \\ ${ }^{(1)}$ Beijing Laboratory of Electron Microscopy, Chinese Academy of Sciences, P.O. Box 2724, \\ Beijing 100080, People's Republic of China \\ ${ }^{(2)}$ Department of Materials Physics, University of Science and Technology Beijing, Beijing 100083, People's Republic of China \\ ${ }^{(3)}$ Institute of Physics, Chinese Academy of Sciences, P.O. Box 603, Beijing 100080, People's Republic of China \\ (Received 28 November 1990)
}

\begin{abstract}
Domains and discommensurations have been directly observed by transmission electron microscopy in some dark-field images using certain reflections in a $\mathrm{Mn}-\mathrm{Si}-\mathrm{Al}$ octagonal quasicrystal. The invisibility and visibility of domains and discommensurations separate the diffraction spots into basic and satellite reflections. The domains and discommensurations observed in satellite reflections are interpreted as due to the ordering of $\mathrm{Si}$ and $\mathrm{Al}$ atoms in the quasilattice of the quasicrystal. A simple model which gives satisfactory agreement with the experimental results is given.
\end{abstract}

PACS numbers: $61.42 .+\mathrm{h}, 61.16 . \mathrm{Di}, 61.50 . \mathrm{Ks}, 61.55 . \mathrm{Hg}$

The discovery of quasicrystals with icosahedral $m 35$ point-group and quasiperiodic translational symmetry in rapidly solidified $\mathrm{Al}-\mathrm{Mn}$ alloys aroused great interest in the study of quasicrystals [1,2]. Quasicrystals with dodecagonal, decagonal, and octagonal symmetry were soon discovered in succession [3-7]. Quasicrystals have now been discovered in many alloy systems. By analogy with incommensurately modulated crystals [8], diffraction peaks of quasicrystals can be indexed as periodic structures in a space with dimensions greater than three [9-11]. In a modulated crystal, diffraction peaks can be separated into two sets, basic reflections and satellite reflections which are usually much weaker than the basic reflections. Furthermore, dark-field images taken with satellite reflections often provide information on the origin of the modulation. Antiphase boundaries in $\mathrm{CuAu}$ II alloy arising from the ordering of atomic species and discommensurations in $\mathrm{TaSe}_{2}$ due to charge-density waves [12-14] are examples of this kind. Reflections of a quasicrystal, however, cannot be separated into basic and satellite reflections. In our study of a $\mathrm{Mn}-\mathrm{Si}-\mathrm{Al}$ octagonal quasicrystal by transmission electron microscopy (TEM), we have directly observed domain structures in some dark-field images using certain reflections. A preliminary analysis of the results is reported here.

An alloy with nominal composition $\mathrm{Mn}_{80} \mathrm{Si}_{15} \mathrm{Al}_{5}$ was obtained by melting high-purity manganese, silicon, and aluminum in argon atmosphere in an arc furnace. Quasicrystal thin films were then prepared by remelting and rapid solidification of the alloy by the piston-and-anvil method as reported previously [7]. Specimens for TEM were prepared by ion milling the thin films. A Philips EM 420 electron microscope was employed for taking electron-diffraction patterns and images.

The Mn-Si-Al octagonal quasicrystal is a two-dimensional quasicrystal with a periodic eightfold axis $(A 8)$ and sixteen orthogonal twofold axes (e.g., $A 2 P$ and $A 2 D)$, as shown in Fig. 1. Dark-field images taken with various reflections in nearly two-beam or systematic many-beam conditions near the eightfold axis (Fig. 2) and other zone axes (Fig. 3) show that there are two kinds of images. Images taken with type- $O$ reflections do not give any useful details [Fig. 2(a)], while images taken with type- $X,-Y$, and $-Z$ reflections show the presence of linear features which are interpreted as domain boundaries [Figs. 2(b), 2(c), and 2(d)]. In the image of a type- $X$ reflection [Fig. $2(b)$ ], dark lines forming four-arm

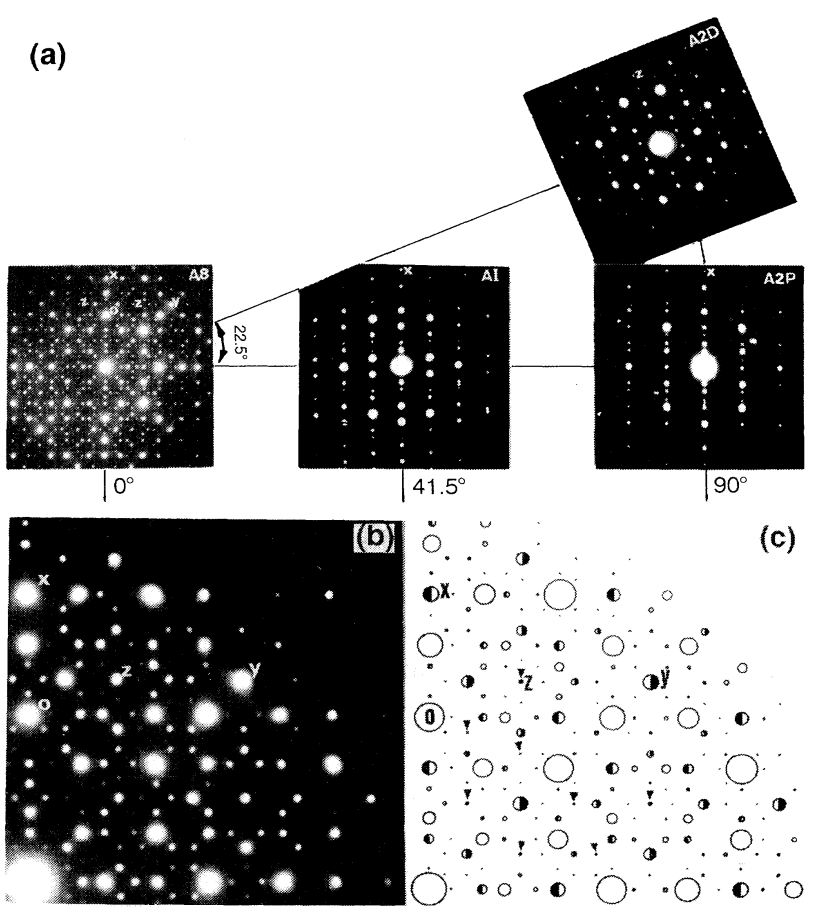

FIG. 1. (a) Electron-diffraction patterns of the Mn-Si-Al octagonal quasicrystal showing the eightfold axis, two twofold axes, and an intermediate axis. The four types of reflections $(O, X, Y$, and $Z)$ are indicated in the (a),(b) experimental and (c) simulated patterns. 


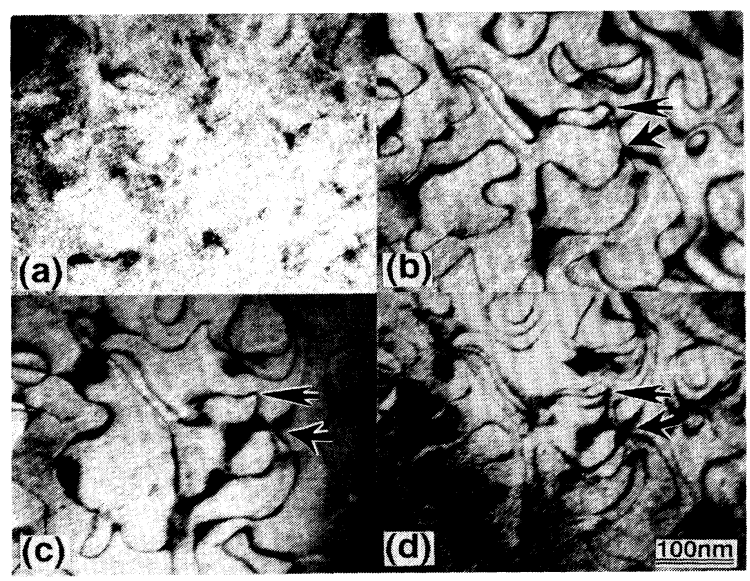

FIG. 2. Dark-field images of (a) type- $O$, (b) type- $X$, (c) type- $Y$, and (d) type- $Z$ reflections near the eightfold axis from the same region. Domain structures are invisible in type- $O$ reflections. Discommensurations (DCs) or domain boundaries are visible in type- $X,-Y$, and $-Z$ images. Note the contrast reversal in (b) and (c). (d) A superposition of (b) and (c). An 8-DC and a 4-DC node are marked by arrows.

nodes and loops are clearly visible. In addition, faint bright lines are also seen in groups of four at the nodes of the dark lines so that the nodes are in fact eight-arm nodes. Faint bright loops are also present. The intersection of dark and bright loops gives rise to four-arm nodes. Thus dark lines alternate with bright lines in groups of four and two. In the image of a type- $Y$ reflection taken from the same area [Fig. 2(c)], the same features are seen but the contrast is reversed. The contrast of all the lines in the image of a type- $Z$ reflection is dark [Fig. $2(\mathrm{~d})]$. The contrast in a $Z$ reflection image may be regarded as a superposition of the contrast of a type- $X$ and a type- $Y$ reflection. This is to be expected since a $Z$ reflection can be obtained as a vector sum of an $X$ and a $Y$ reflection. Note that the intensity of the bright lines, which is weak, decreases rapidly with increasing thickness, so that they become invisible in thicker specimens. Images from the $A 2 D$ and $A I$ zone axes are shown in Figs. 3(a) and 3(b). It is clear from Fig. 1 that only type- $Z$ and type- $X$ images can be obtained near the $A 2 D$ and $A I$ zone axes, respectively. Again dark or darkbright lines intersecting in eight-arm and four-arm nodes are clearly visible. The density of the lines is visibly higher. This difference is not related to the change in orientation. Indeed, there is no change in the discommensuration (DC) spacing when the specimen is tilted through $90^{\circ}$ from the eightfold axis to a twofold axis since the tilt axis is normal to the DC line spacing. Image details in the tilt direction are of course foreshortened. The images in Figs. 2, 3(a), and 3(b) have been taken from different specimens. We have also found that different regions of the same specimen give different densities. Changing the diffraction condition from systemat-

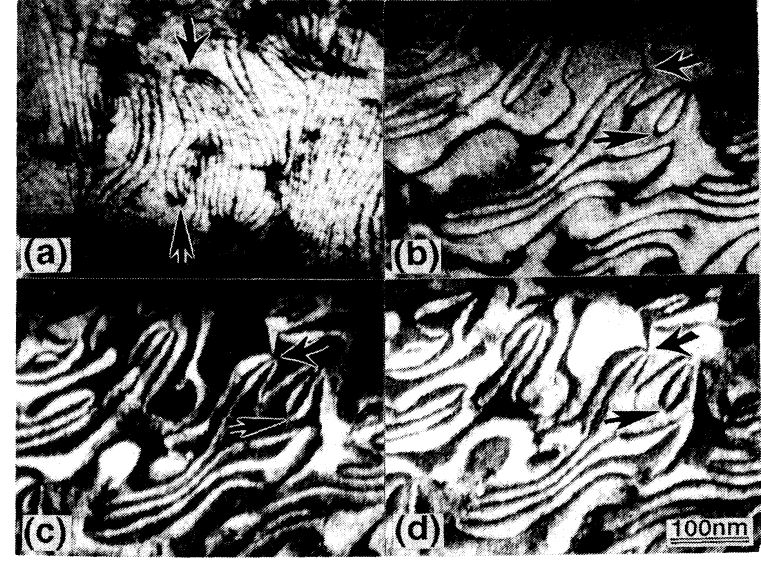

FIG. 3. Dark-field images of (a) a type- $Z$ reflection near the $A 2 D$ axis and (b) a type- $X$ reflection near the $A I$ axis showing domain-boundary contrast. Changing the diffraction from systematic row to nearly axial cross grating, domain contrast is obtained in (c) and (d). Contrast reversal in (c) and (d) is due to the reversal of diffraction vector. 8-DC and 4-DC nodes are marked by arrows.

ic row to near axial cross grating, dark and bright domain contrast [Figs. 3(c) and 3(d)] instead of dark and bright domain-boundary contrast [Fig. 3(b)] is obtained. The domain contrast is reversed when the diffraction vector is reversed [Figs. 3(c) and 3(d)]. This is similar to the characteristics of inversion domains in a $\chi$ phase [15]. Four or two dark domains alternate with bright domains resulting in domain clusters of eight or four at a point. Typically, the width of a domain, or the separation between domain boundaries, varies between 10 and $30 \mathrm{~nm}$ (Figs. 2 and 3).

The domain boundaries in Figs. 2 and 3 terminating in groups of four and eight are discommensurations [16]. There are two kinds of DCs, showing, respectively, dark and bright contrast. The node where DCs terminate is termed a dislocation. The phase change around a DC dislocation must be a multiple of $2 \pi$. Similar DC configurations have been observed in incommensurate crystals, for instance, $\mathrm{TaSe}_{2}, \mathrm{Ag}-\mathrm{Mg}$ alloys, and $\mathrm{Rb}_{2} \mathrm{ZnCl}_{4}$ $[13,14,17,18]$. Two kinds of DCs have also been observed in $\mathrm{Rb}_{2} \mathrm{ZnCl}_{4}$ [18]. The variation of the DC density in Figs. 2 and 3 is interpreted as different frozen-in states in the commensuration process due to the different rates of cooling. The commensuration process is expected to take place by the elimination of DCs through the motion of the DC dislocations $[13,14,17,18]$. Taking a closed circuit about an 8-DC node, the change of phase across each DC is $2 \pi n / 8$ for $n=1$ or $\pi / 4$. This is also the angular separation between type- $X$ and type- $Y$ reflections. Similarly, taking a circuit about a 4-DC node, the change of phase across each $D C$ is $\pi / 2$.

In the eightfold electron-diffraction pattern, type- $O$ reflections in which the domain structures are invisible 

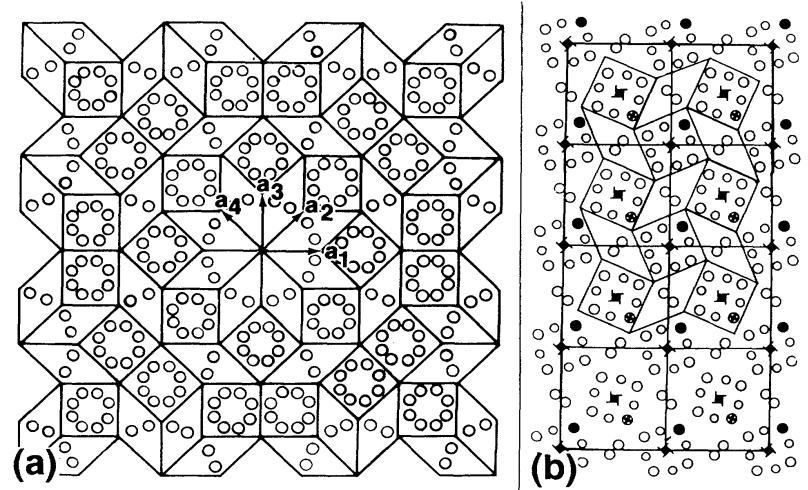

FIG. 4. (a) A simple model of the octagonal quasicrystal obtained by decorating the octagonal quasilattice with eight $\mathrm{Mn}$ atoms at the centers of the squares and two $\mathrm{Si}$ or $\mathrm{Al}$ atoms at the centers of the $45^{\circ}$ rhombi. (b) [001] projection of $\beta$ - Mn structure showing a periodic array of squares with eight atoms and $45^{\circ}$ rhombi with two atoms.

form an octagonal quasicrystal lattice [open circles in Fig. 1(c)]. This quasicrystal lattice can be regarded as a basic quasilattice while type- $X,-Y$, and $-Z$ reflections in which the domain structures are visible can be regarded as satellite reflections arising from the ordering of specific chemical species in the basic octagonal quasicrystal lattice. Type- $X$ reflections correspond to diffraction from one set of domains and type- $Y$ reflections correspond to diffraction from a $45^{\circ}$-rotated set of domains. Type- $Z$ reflections are common to both sets of domains. A simple model for the domain structures due to ordering in the octagonal quasicrystal lattice is proposed as follows.

The octagonal phase is a two-dimensional (2D) quasicrystal with periodicity along the eightfold axis and quasiperiodicity in planes normal to the eightfold axis. The $2 \mathrm{D}$ quasiperiodic lattice can be generated by squares and $45^{\circ}$ rhombi in a definite fashion. Any vector in the quasilattice can be expressed as a linear combination of the set of four basis vectors:

$$
\mathbf{a}_{i+1}=R \mathbf{a}_{i}, \quad i=1,2,3,
$$

where $R$ is a rotation operation of $\pi / 4$ parallel to the eightfold axis normal to the $2 \mathrm{D}$ quasilattice plane. The octagonal quasilattice can also be obtained by projecting a 4D cubic lattice onto a 2D hyperplane [19]. The squares in the 2D quasilattice are denoted by two orthogonal vectors: $\left(\mathbf{a}_{1}, \mathbf{a}_{3}\right)$ and $\left(\mathbf{a}_{2}, \mathbf{a}_{4}\right)$. The $45^{\circ}$ rhombi are denoted by $\left(\mathbf{a}_{1}, \mathbf{a}_{2}\right),\left(\mathbf{a}_{2}, \mathbf{a}_{3}\right),\left(\mathbf{a}_{3}, \mathbf{a}_{4}\right)$, and $\left(\mathbf{a}_{1}, \mathbf{a}_{4}\right)$. The octagonal quasicrystal can be obtained by decorating the squares and rhombi with $\mathrm{Mn}$ and $\mathrm{Si}$ or $\mathrm{Al}$ atoms, respectively, as shown in Fig. 4(a). The atoms are shown symmetrically placed with respect to the respective centers. The atomic positions in the $4 \mathrm{D}$ cubic cell are as

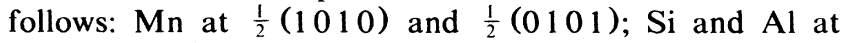
$\frac{1}{2}(1100), \frac{1}{2}(0110), \frac{1}{2}(0011)$, and $\frac{1}{2}(1001)$. The eight $\mathrm{Mn}$ atoms and two $\mathrm{Si}$ or $\mathrm{Al}$ atoms are placed at the respective center-of-mass positions. The diffraction pattern of this decoration is shown in Fig. 1(c). It can be seen that the calculated pattern matches the experimental pattern quite well, better than the pattern calculated from a decoration based on atoms placed at the quasilattice points [19]. This decoration is based on the arrangement of atoms in $\beta$ - $\mathrm{Mn}$ (space group $P 4,32, a=0.63 \mathrm{~nm}$ ) [Fig. 4(b)] [20]. In a [001] projection, the $\beta-\mathrm{Mn}$ structure is a periodic arrangement of squares and $45^{\circ}$ rhombi with eight and two atoms, respectively. The arrangement of squares and $45^{\circ}$ rhombi in the octagonal quasicrystal lattice is quasiperiodic. In a previous investigation, we have found that the octagonal quasicrystal coexists with $\beta$-Mn-type crystal [7]. The transformation of the octagonal quasicrystal to $\beta$-Mn-type crystal structure has been observed and interpreted as due to the gradual introduction of phason strain [21]. Consider one kind of ordering in which an $\mathrm{Al}$ atom occupies the position $\frac{1}{2}\left(\begin{array}{lll}1 & 100)\end{array}\right)$ and $\mathrm{Si}$ atoms occupy positions $\frac{1}{2}\left(\begin{array}{llll}0 & 1 & 1 & 0\end{array}\right), \frac{1}{2}\left(\begin{array}{llll}0 & 0 & 1 & 1\end{array}\right)$, and

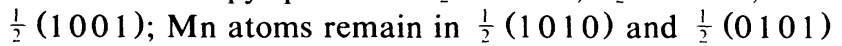
positions. Since the rhombus positions are equivalent, $\mathrm{Al}$ atoms can occupy each of the four rhombus positions, giving rise to four types of domains:

\begin{tabular}{|c|c|c|}
\hline Al position & Si position & Domain \\
\hline$\frac{1}{2}\left(\begin{array}{llll}1 & 1 & 0 & 0\end{array}\right)$ & $\frac{1}{2}\left(\begin{array}{llll}0 & 1 & 1 & 0\end{array}\right), \frac{1}{2}\left(\begin{array}{llll}0 & 0 & 1 & 1\end{array}\right), \frac{1}{2}\left(\begin{array}{lllll}1 & 0 & 0 & 1\end{array}\right)$ & I \\
\hline$\frac{i}{2}\left(\begin{array}{llll}0 & 1 & 1 & 0\end{array}\right)$ & 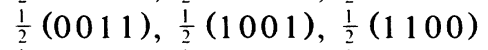 & II \\
\hline$\frac{1}{2}(0011)$ & $\frac{1}{2}\left(\begin{array}{llll}0 & 0 & 0 & 1\end{array}\right), \frac{1}{2}\left(\begin{array}{llll}1 & 1 & 0 & 0\end{array}\right), \frac{1}{2}\left(\begin{array}{llll}0 & 1 & 1 & 0\end{array}\right)$ & III \\
\hline$\frac{1}{2}(10001)$ & 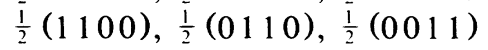 & IV \\
\hline
\end{tabular}

Even-odd domains are related by a rotation of $\pi / 4$. Even-even and odd-odd domains are related by a rotation of $\pi / 2$. The occurrence of both eight and four clusters of domains and DCs in the Mn-Si-Al octagonal quasicrystal can be understood in terms of the grouping of even-odd domains and even-even (or odd-odd) domains. The interpretation of the domain walls or DCs in terms of ordering of $\mathrm{Si}$ and $\mathrm{Al}$ atoms in the octagonal quasilattice is consistent with the existence of domain walls and phason strains in the random tiling model of the two-dimensional quasicrystal of Li, Park, and Widom [22].

Recently, antiphase domain structures have been observed in the newly discovered icosahedral quasicrystals in $\mathrm{Al}-\mathrm{Cu}-\mathrm{Fe}$ and related ternary systems $[23,24]$. The antiphase domains have also been interpreted as arising from the ordering of atoms. It is not clear if the domains are introduced and removed by the motion of DC dislocations [16]. It would be interesting to study the motion of $\mathrm{DC}$ dislocations in the $\mathrm{Mn}-\mathrm{Si}-\mathrm{Al}$ octagonal quasicrystal. The different frozen-in states of DCs in Figs. 2 and 3 lend support to the removal of DCs by motion of DC dislocations. More recently, in the study of the Mo-Cr-Ni octagonal quasicrystalline system [25], phason-strained imperfect diffraction patterns have been observed in which the diffraction spots corresponding to the $O$ spots and $X$, $Y$, and $Z$ spots in the $\mathrm{Mn}-\mathrm{Si}-\mathrm{Al}$ system are shifted and 
split, respectively. DCs of much higher density are observed in the dark field taken with the split spots. More systematic study is currently under way.

In summary, we have shown that domains and DCs can be directly observed by TEM in some reflections in the Mn-Si-Al octagonal quasicrystal. Based on the invisibility and visibility of the domain structures, basic and satellite reflections can be identified. The domains and DCs can be satisfactorily interpreted with a simple model based on the ordering of $\mathrm{Si}$ and $\mathrm{Al}$ atoms in the basic quasilattice.

The authors would like to thank J. Q. You for help in computing work. The work reported here is supported in part by the National Natural Science Foundation of China.

[1] D. Shechtman, I. Blech, D. Gratias, and J. W. Cahn, Phys. Rev. Lett. 53, 1951 (1984).

[2] D. Levine and P. J. Steinhardt, Phys. Rev. Lett. 53, 2477 (1984).

[3] T. Ishimasa, H. U. Nissen, and Y. Fukano, Phys. Rev. Lett. 55, 511 (1985).

[4] H. Chen, D. X. Li, and K. H. Kuo, Phys. Rev. Lett. 60, 1645 (1988).

[5] L. Bendersky, Phys. Rev. Lett. 55, 1461 (1985).

[6] K. K. Fung, C. Y. Yang, Y. Q. Zhou, J. G. Zhao, W. S. Zhan, and B. G. Shen, Phys. Rev. Lett. 56, 2060 (1986).

[7] N. Wang, H. Chen, and K. H. Kuo, Phys. Rev. Lett. 59, 1010 (1987).

[8] See, for instance, T. Janssen and A. Janner, Adv. Phys.
36, 519 (1987).

[9] P. A. Kalugin, A. Yu. Kitaev, and L. S. Levitov, Pis'ma Zh. Eksp. Teor. Fiz. 41, 119 (1985) [JETP Lett. 41, 145 (1985)].

[10] M. Duneau and A. Katz, Phys. Rev. Lett. 54, 2688 (1985).

[11] V. Elser, Acta Crystallogr. A 42, 36 (1986).

[12] D. W. Pashley and A. E. B. Presland, J. Inst. Met. 87, 419 (1958).

[13] K. K. Fung, S. McKernan, J. W. Steeds, and J. A. Wilson, J. Phys. C 14, 5417 (1981).

[14] C. H. Chen, J. M. Gibson, and R. M. Fleming, Phys. Rev. B 26, 184 (1982).

[15] R. Serneels, M. Snykers, P. Delavignette, R. Gevers, and S. Amelinckx, Phys. Status Solidi (b) 58, 277 (1973).

[16] W. L. McMillan, Phys. Rev. B 14, 1496 (1976).

[17] Y. Fujino, H. Sato, M. Hirabayashi, E Aoyagi, and Y. Koyama, Phys. Rev. Lett. 58, 1012 (1987).

[18] K. Tsuda, N. Yamamoto, and K. Yaki, Acta Crystallogr. A 44, 864 (1988).

[19] Z. M. Wang and K. H. Kuo, Acta Crystallogr. A 44, 857 (1988).

[20] N. Wang and K. H. Kuo, Philos. Mag. B 60, 347 (1989), and references therein.

[21] Z. H. Mai, X. Lu, N. Wang, K. H. Kuo, Z. C. Jin, and G. Cheng, Phys. Rev. B 40, 12183 (1989).

[22] W. Li, H. Park, and M. Widom, J. Phys. A 23, L573 (1990); (to be published).

[23] J. Devaud-Rzepski, A. Quivy, Y. Calvayrac, and M. Cornier-Quiquandon, Philos. Mag. B 60, 855 (1989).

[24] S. Ebalard and F. Spaepen, J. Mater. Res. 5, 62 (1990).

[25] H. Chen, Y. He, S. Burkov, S. J. Poon, and G. J. Shiflet, Bull. Am. Phys. Soc. 35, 522 (1990). 


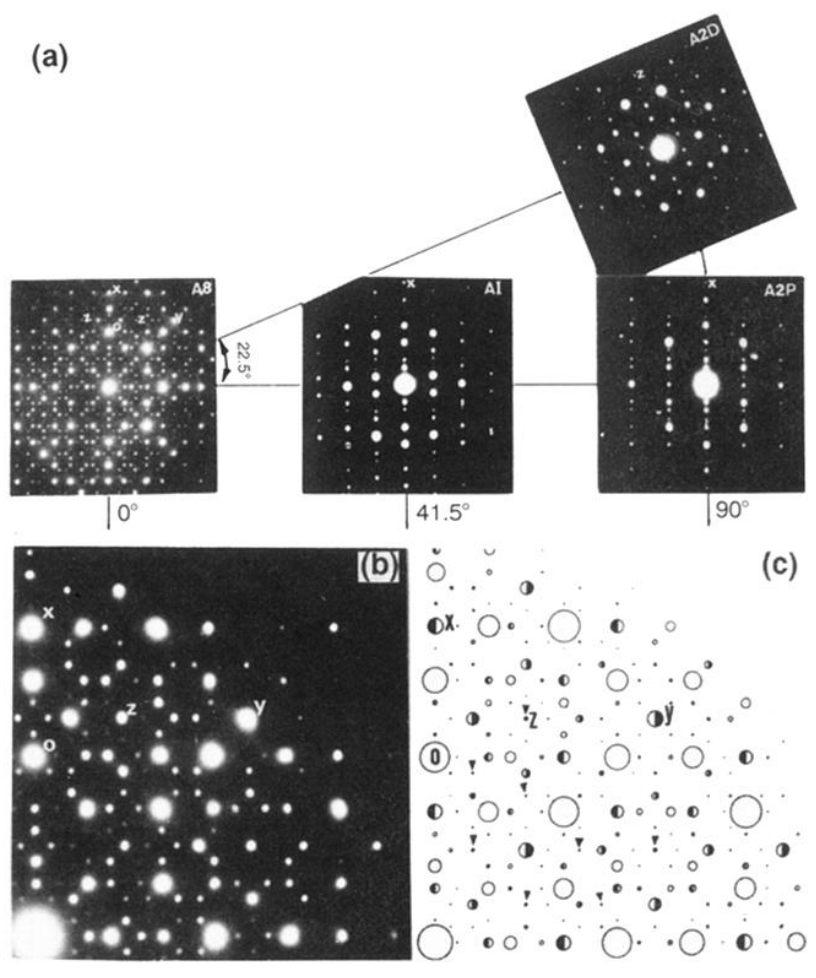

FIG. 1. (a) Electron-diffraction patterns of the Mn-Si-Al octagonal quasicrystal showing the eightfold axis, two twofold axes, and an intermediate axis. The four types of reflections $(O, X, Y$, and $Z)$ are indicated in the (a),(b) experimental and (c) simulated patterns. 


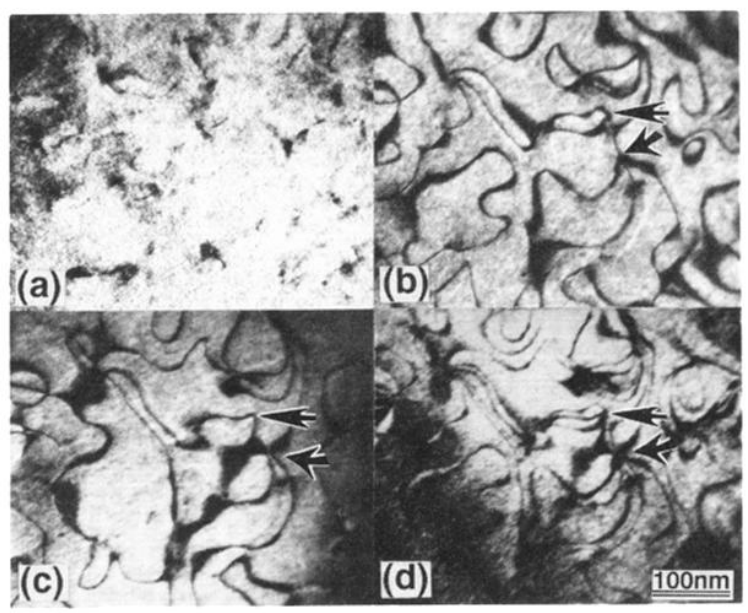

FIG. 2. Dark-field images of (a) type- $O$, (b) type- $X$, (c) type- $Y$, and (d) type- $Z$ reflections near the eightfold axis from the same region. Domain structures are invisible in type- $O$ reflections. Discommensurations (DCs) or domain boundaries are visible in type- $X,-Y$, and $-Z$ images. Note the contrast reversal in (b) and (c). (d) A superposition of (b) and (c). An 8-DC and a 4-DC node are marked by arrows. 


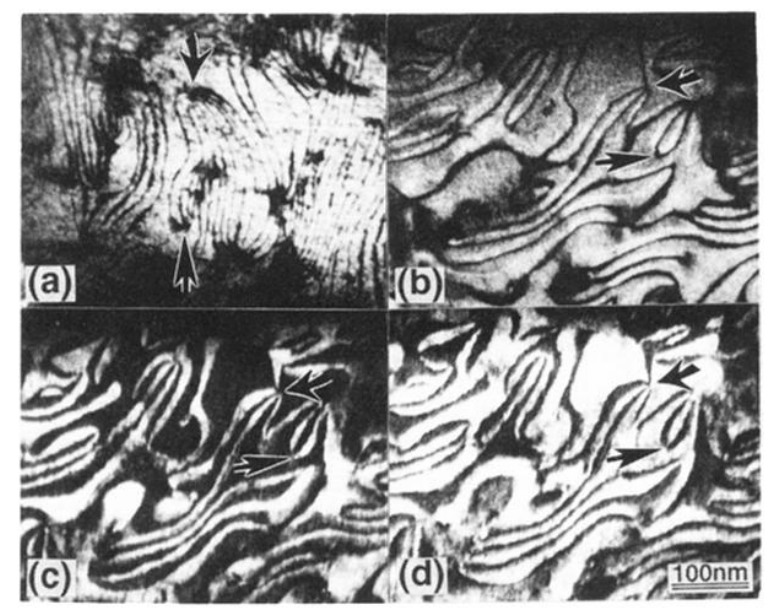

FIG. 3. Dark-field images of (a) a type- $Z$ reflection near the $A 2 D$ axis and (b) a type- $X$ reflection near the $A I$ axis showing domain-boundary contrast. Changing the diffraction from systematic row to nearly axial cross grating, domain contrast is obtained in (c) and (d). Contrast reversal in (c) and (d) is due to the reversal of diffraction vector. 8-DC and 4-DC nodes are marked by arrows. 